\title{
Erratum: Measurement and Simulation of Stilbene Scintillator Response for the KSTAR Neutron Diagnostic System
}

\author{
[J. Korean Phys. Soc. 64, 645 (2014)] \\ Seung Kyu LeE \\ Korea Atomic Energy Research Institute, 989-111 Daedeok-daero, Yuseong, Daejeon 305-353, Korea, \\ and Department of Nuclear Engineering, Hanyang University, Seoul 133-791, Korea \\ Jae Bum Son and Jeong Soo KAng \\ Department of Nuclear Engineering, Hanyang University, Seoul 133-791, Korea \\ Hee Seo, Byung-Hee Won, Se-Hwan PARK and Ho-Dong Kim \\ Korea Atomic Energy Research Institute, Daejeon 305-353, Korea \\ Byoung Hwi Kang and Gi Dong Kim \\ Institute for Basic Science, Daejeon 305-811, Korea \\ Yong Kyun Kiм* \\ Department of Nuclear Engineering, Hanyang University, Seoul 133-791, Korea, \\ and Institute for Basic Science, Daejeon 305-811, Korea
}

DOI: $10.3938 /$ jkps.64.1234

Table 1 should be included in this paper by the following table.

Table 1. General properties of the stilbene scintillator.

\begin{tabular}{cc}
\hline \hline Property & Value \\
\hline Light output $(\%$ anthracene) & 50 \\
\hline Density $\left(\mathrm{g} / \mathrm{cm}^{3}\right)$ & 1.22 \\
\hline H/C ratio & 0.857 \\
\hline Decay time $(\mathrm{ns})$ & 3.5 \\
\hline Luminescence max $(\mathrm{nm})$ & 390 \\
\hline Hygroscopy & No \\
\hline \hline
\end{tabular}

*E-mail: ykkim4@hanyang.ac.kr; Fax: +82-2-2296-2354 